\title{
Surgical Complications of Presurgical Systemic Therapy for Renal Cell Carcinoma: A Systematic Review
}

\author{
Barrett McCormick, Matthew A. Meissner, Jose A. Karam and Christopher G. Wood* \\ The University of Texas MD Anderson Cancer Center, Houston, TX, USA
}

\begin{abstract}
.
Background: Locally advanced and metastatic renal cell carcinoma (RCC) is associated with poor survival outcomes. The integration of presurgical systemic therapy with targeted molecular agents prior to surgical resection of RCC tumors has been utilized to improve on these outcomes. These agents may be associated with an increased risk of perioperative complications due to their action on angiogenesis and cell proliferation.

Objective: To examine the evidence for the incidence and severity of perioperative complications following presurgical targeted therapy for locally advanced or metastatic RCC.

Methods: We performed a systematic review of retrospective studies, prospective clinical trials, and meta-analyses using key search terms in PubMed and Medline. Studies were screened for eligibility and data were extracted by the authors. A qualitative analysis was performed and the complications for available targeted agents was reported.

Results: Retrospective analyses and small prospective trials indicate varying complication rates and types based on presurgical therapies. While some studies indicate a possible increase in wound-related complications, other studies did not show similar results. Additional unique complications reported include an increase in surgical adhesions. There was not any significant difference in overall or bleeding complications.

Conclusions: Overall, these studies demonstrate an acceptable level of surgical complications that should not discourage the clinician considering presurgical therapy. The results of pending trials looking at presurgical therapies will provide further information.
\end{abstract}

Keywords: Renal cell carcinoma, pre-surgical therapy, targeted therapy, complications

\section{INTRODUCTION}

Kidney cancer represents a growing problem in the United States with an estimated 63,990 new cases of the disease in 2017 and is projected to

${ }^{*}$ Correspondence to: Christopher G. Wood, M. D., FACS, Professor and Deputy Chairman, Douglas E. Johnson, M. D. Endowed Professorship in Urology, Department of Urology, The University of Texas MD Anderson Cancer Center, 1515 Holcombe Blvd. Unit 1373, Houston, TX 77030, USA. Tel.: +1 713563 7463; Fax: +1 713792 3474; E-mail: cgwood@mdanderson.org. account for over 14,000 deaths [1]. Largely due to the detection of small renal tumors from the increased utilization of cross-sectional imaging, the majority of patients present with localized disease. However, nearly $20 \%$ still present with locally advanced or distant metastatic disease [2]. Survival outcomes following surgery alone for locally advanced disease are suboptimal, with a 5-year progression free survival probability of only $30 \%$ for patients with $\mathrm{T} 4$ disease or pathologic lymph node involvement [3]. Improving on these outcomes remains a clinical challenge. 
A greater understanding of the mechanisms of RCC carcinogenesis has facilitated the development of targeted molecular therapies for RCC over the past decade. These new agents inhibit specific components of the vascular endothelial growth factor receptor (VEGFR), platelet derived growth factor receptor (PDGFR), mammalian target of rapamycin (mTOR), and down-stream signaling pathways involved in angiogenesis and metabolism for RCC [4]. More recently, immune checkpoint inhibition with monoclonal antibodies against PD-1/PD-L1 has shown to improve survival in patients with metastatic RCC both as first-line treatment, and in those who previously failed second-line therapy with signal transduction inhibitors $[5,6]$.

Despite these advances, surgical resection of the primary tumor remains a key component in the management of advanced RCC. Two landmark clinical trials, conducted in the cytokine therapy era, found that cytoreductive nephrectomy $(\mathrm{CN})$ conferred a modest survival benefit for patients with metastatic RCC and good performance status [7, 8]. Current guidelines have extrapolated this concept to the targeted therapy era, and recommend $\mathrm{CN}$ in selected patients [9]. A recent analysis from the National Cancer Database evaluated the utilization and survival of $\mathrm{CN}$ in patients who received targeted therapy. This analysis found that $\mathrm{CN}$ correlated with improved overall survival compared to no $\mathrm{CN}$ [10]. However, for patients with high-risk, non-metastatic RCC, the most effective integration of targeted therapy remains unclear in regards to the proper agent, dose, and timing. Notably, two phase III clinical trials examining TKIs in the adjuvant setting have produced conflicting results for disease-free survival [11-13].

"Presurgical" or "preoperative" therapy with targeted agents in patients with clinical M0 or M1 disease has been investigated in small prospective trials $[14,15]$. The purported benefits of presurgical therapy include eradication of micrometastatic disease, tumor down-staging, and easing surgical resectability [16]. One major concern with presurgical therapy is the potential to increase intra-operative and post-operative complications. Experience with traditional, cytotoxic chemotherapy for other GU malignancies suggests that therapy in the neoadjuvant setting does not significantly increase perioperative complications, or the difficulty of surgical resection [17]. The therapies targeting the VEGF axis act specifically on pro-angiogenic pathways to inhibit RCC tumor growth, but also interfere with normal wound healing, tissue oxygen tension, and formation of neovascularity [18]. Investigators have observed an increase in wound healing and thromboembolic complications following nephrectomy in patients treated with targeted agents [19].

In this systematic review, we examine the incidence and severity of surgical complications in patients undergoing surgery for RCC who were treated with presurgical therapy.

\section{METHODS}

We performed a PubMed and Medline search of English-language articles from inception until $7 / 2017$. Key search terms included renal cell carcinoma, kidney cancer, nephrectomy, neoadjuvant therapy, perioperative therapy, targeted therapy, and surgical complications. We searched full-text articles for prospective clinical trials, retrospective studies, systematic reviews, and meta-analyses. Reports were individually reviewed and data extracted by the authors. We excluded case reports, preclinical studies, studies which did not report on complications, and studies in which patients did not undergo surgery.

\section{RESULTS}

Initial search yielded 79 records, and after duplicate records were removed, a total of 47 records were screened for eligibility. An additional 21 records were excluded and therefore the final qualitative analysis included 16 records. Figure 1 shows the flow diagram detailing the studies screened, assessed for eligibility, records excluded, and the number of studies included in the final qualitative evidence synthesis. Table 1 summarizes the prospective studies that reported perioperative complications in patients who received presurgical therapy.

\section{COMPLICATIONS OF SPECIFIC TARGETED THERAPY AGENTS}

\section{Bevacizumab}

Bevacizumab is a monoclonal antibody directed against VEGF that inhibits angiogenesis in tumor cells. The adverse event profile includes hypertension, proteinuria, and impaired wound healing [20]. It has shown to be effective in improving progressionfree survival in combination with interferonalpha immunotherapy compared to interferon-alpha 


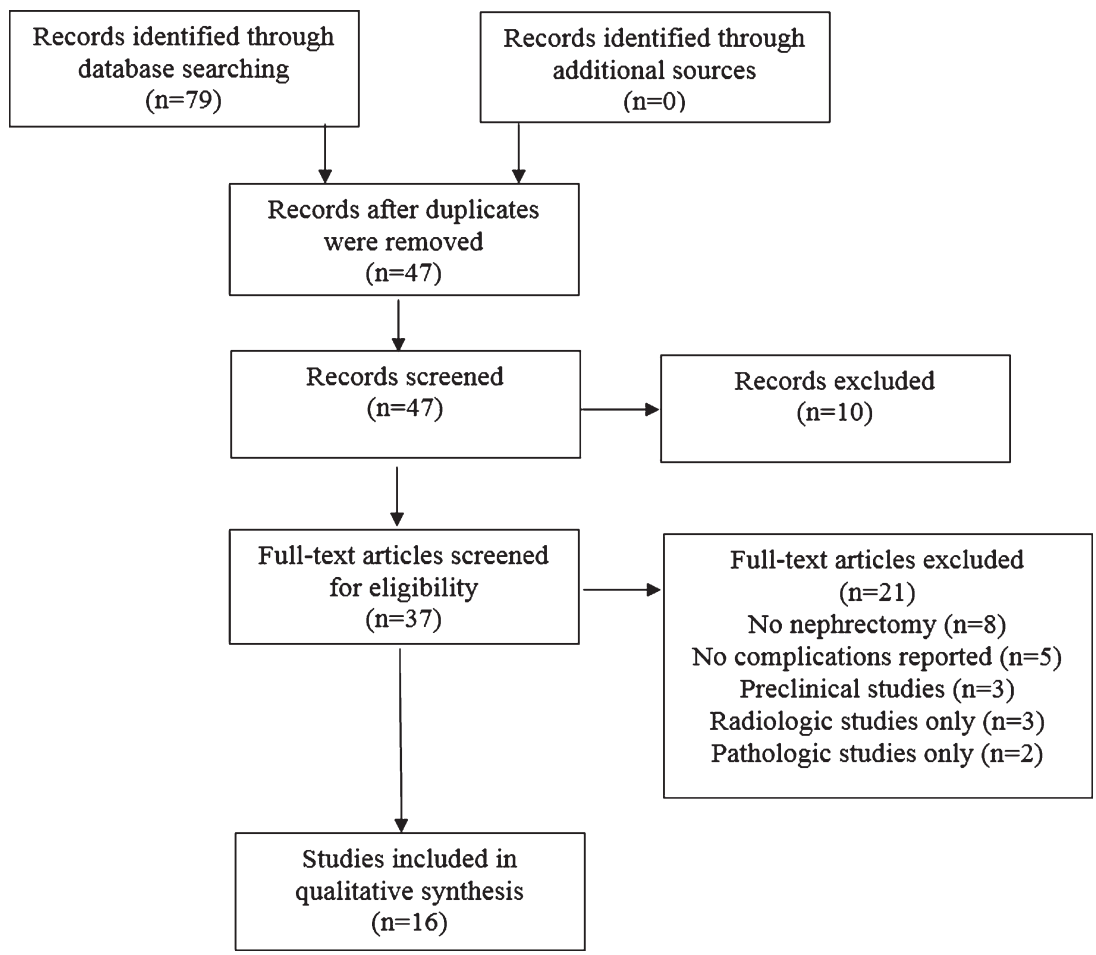

Fig. 1. Flow diagram for litrature search and screening for eligible studies included in the systematic review.

Table 1

Prospective studies of presurgical targeted therapy for RCC reporting perioperative complications

\begin{tabular}{|c|c|c|c|c|c|c|c|}
\hline & \multirow{2}{*}{$\begin{array}{l}\text { Presurgical } \\
\text { Agent }\end{array}$} & \multirow{2}{*}{$\begin{array}{l}\text { Duration } \\
\text { of Therapy } \\
\text { (weeks) }\end{array}$} & \multirow{2}{*}{$\begin{array}{l}\text { No. Patients } \\
\text { undergoing } \\
\text { Surgery (n) }\end{array}$} & \multicolumn{4}{|c|}{ Complication No. $(\%)$} \\
\hline & & & & $\begin{array}{l}\text { Wound- } \\
\text { healing }\end{array}$ & Thromboembolic & Bleeding & Other \\
\hline Jonasch et al. & Bevacizumab & 8 & 42 & $\begin{array}{l}9(20.9) \\
\text { fascial dehiscence }=1\end{array}$ & $1(0.05)$ & 0 & \\
\hline Cowey et al. & Sorafenib & 4.5 (median) & 30 & 0 & 0 & 0 & \\
\hline Powles et al & Sunitinib & $8-12$ & 47 & $6(9)$ & 0 & $1(2)$ & Renal failure 2 [4] \\
\hline Rini et al. & Sunitinib & 12 & 29 & 0 & 0 & 0 & $\begin{array}{l}\text { Pulmonary edema } 1 \\
\text { Acute renal failure } 2[6]\end{array}$ \\
\hline Karam et al. & Axitinib & 12 & 24 & $1(4.2 \%)$ & $2(8.3)$ & $1(4.2)$ & $\begin{array}{c}\text { Chylous ascites } 3 \text { (12.5) } \\
\text { Gastroparesis } 1(4.2)\end{array}$ \\
\hline Rini et al. & Pazopinib & 16 & 25 & $2(8)$ & 0 & $1(5)$ & Urine leak 5 [25] \\
\hline
\end{tabular}

therapy alone for the treatment of metastatic RCC $[21,22]$. Bevacizumab in combination with interferon remains a first-line treatment option for metastatic RCC.

The feasibility of safely performing cytoreductive nephrectomy following treatment with bevacizumab was investigated by Jonasch et al. in a single-arm, phase II study [14]. Of the 50 patients in the study, $84 \%$ were able to undergo nephrectomy after completion of preoperative therapy, while $12 \%$ had disease progression or deterioration of performance status. Bevacizumab was withheld for 4 weeks preceding nephrectomy. The most notable complication was a $20.9 \%$ incidence of delayed wound healing postoperatively. This included one fascial dehiscence requiring operative intervention, and 3 patients who required discontinuation of therapy. Although the study did not include a control arm, the authors compared their findings to a matched, historical cohort and found the rate of delayed wound healing to be significantly higher in patients who received presurgical bevacizumab $(20.9 \%$ v $2 . \%, p<0.001)[14]$. Of note, the first 23 patients enrolled in the trial received oral erlotinib in addition to bevacizumab. Erlotinib was removed from the protocol after phase II data emerged showing a lack of efficacy in RCC. 
The concerns about wound healing have been noted following treatment with bevacizumab for nonrenal malignancies. A recent meta-analysis of 7 randomized-controlled trials examined the incidence of wound-healing complications following therapy for gastrointestinal, breast, lung and renal malignancies [23]. The pooled analysis revealed a two-fold increase in the risk of delayed wound-healing or wound complications for patients receiving bevacizumab [OR 2.32, 95\% CI 1.43-3.75] [23]. These results, however, were heavily weighted by the large number of patients undergoing treatment for advanced colorectal cancer [23].

With the advent of newer targeted therapies as well as immune checkpoint inhibitors, bevacizumab is likely to have a limited role in the presurgical setting for RCC.

\section{Signal transduction inhibitors}

The advent of signal transduction inhibitors in the treatment of RCC started a promising new strategy in the management of advanced disease. Signal transduction is a complex process involving numerous different pathways mentioned previously in this review. RCC has been noted to be specifically suited for targeting of these inhibitors; particularly of the kinase pathways involved in tumor growth and angiogenesis [24]. The side effect profile commonly associated with the tyrosine kinase inhibition includes hypertension, hematological abnormalities, fatigue, stomatitis, and hand-foot syndrome. However, these adverse effects are generally considered manageable with a low overall incidence of serious problems [25].

\section{Sunitinib}

Sunitinib is an oral tyrosine kinase inhibitor which exhibits anti-tumor activity via targeting of numerous receptors, mainly VEFGR. Numerous studies have evaluated its efficacy and complication profile when given as presurgical therapy in the context of larger studies looking at numerous agents, or those looking at sunitinib alone. Chapin et al. retrospectively evaluated a cohort of 70 patients who received various agents prior to $\mathrm{CN}$. While the majority of their study patients received single or combination therapy with bevacizumab, 14 patients received single agent sunitinib [19]. Overall, this study identified that complications greater than ninety days post-operatively were more likely in patients who had received presurgical therapy (15.9\% vs $3.8 \%$; $p=0.002$ ) [19]. Additionally, those patients who did receive presurgical therapy and experienced a complication were more likely to have multiple events (76\% vs 51\%; $p=0.013$ ). As mentioned previously, this study also noted an increased rate of wound related complications. However, the therapy was not an independent predictor for overall complication risk or significant (Clavien $>3$ ) complications. Harshman et al. evaluated a small cohort of patients receiving either sunitinib or sorafenib preoperatively. $70 \%$ of their patients received sunitinib. Wound healing issues were also observed, but not appreciated to be statistically significant $(p=0.55)$ [26]. They also did not appreciate an overall increase in the perioperative complication rate on univariate or multivariate analysis $(p=0.47$ and $p=0.25)$. Interestingly, they did note an increase in the incidence and severity of intraoperative adhesions as measured by a standardized grading system [27]. This clearly could present clinical implications and was believed by the authors to be directly related to the degree of therapeutic shrinkage after presurgical therapy [26].

The safety of presurgical sunitinib therapy has been evaluated in several prospective studies. Hellenthal et al. examined the safety of 90 days of pre-operative sunitinib in 20 patients with clinical T1b-T3 clear cell RCC [28]. Sunitinib therapy was held 5 days prior to surgery for the first 5 patients enrolled, and 24 hours prior to surgery for the remaining patients. No patients experienced any adverse intra-, or postoperative complications due to sunitinib therapy, and routine surgical parameters did not differ significantly when compared to historical controls [28]. Powles et al. reported a combined analysis of two single-arm phase II studies evaluating either two or three cycles of sunitinib prior to nephrectomy with a total cohort of 66 patients. They noted an overall complication rate of $27 \%$ including 5 patients with delayed wound healing and a total of four Clavien 3 or higher complications, including one post-operative death [29]. They did not appreciate any significant differences in complications between those receiving two or three cycles of presurgical therapy, but did note that in those receiving three cycles there was a more prominent incidence of peritumoral fibrosis.

A recent study by Lane et al. assessed as a primary endpoint whether presurgical sunitinib therapy would facilitate partial nephrectomy by sufficiently downsizing the primary tumor. In their study, $86 \%$ of patients ultimately underwent surgical intervention with a $29 \%$ overall complication rate including five 
grade 3 or higher complications [30]. However, the authors did not feel that these complications could be directly attributed to the presurgical use of sunitinib.

\section{Sorafenib}

Sorafenib is an oral tyrosine kinase inhibitor with primary inhibition of the Ras/Raf pathway which has also been noted to be involved in other mechanisms, namely VEGF and PDGF [24].

Cowey et al. performed a prospective study to evaluate the safety and efficacy of presurgical sorafenib. A total of 30 patients underwent sorafenib therapy for a median of 33 days. In their cohort, they observed no complications related to delayed wound healing or surgical dehiscence aside from a single case of a superficial wound separation managed conservatively [31]. These results are consistent with a 2015 study by Zhang et al. which evaluated 18 patients receiving sorafenib prior to surgical intervention. Although this was a smaller cohort of patients, there were no surgical wound healing complications appreciated. One patient who had a concurrent inferior vena cava thrombectomy did require reoperation due to bleeding, but this was not believed to be a result of the presurgical therapy [32].

\section{Pazopanib}

Pazopanib represents an additional novel tyrosine kinase inhibitor targeting the VEGF, PDGF, and c-kit pathways [24]. Powles et al. prospectively evaluated 104 patients with advanced disease who would receive presurgical pazopanib followed by $\mathrm{CN}$ and continued pazopanib after surgery. Although the primary endpoint was the assessment of clinical benefit via RECIST, additional information was obtained regarding survival and surgical complications [33]. A total of 63 patients ultimately underwent surgery. Of these, complications were noted in $22 \%$ of patients with $3 \%$ Grade 3 or higher. One surgical-related death was reported. Overall, the results of this study demonstrated the safety and feasibility of presurgical pazopanib with an acceptable level of surgical complications [33].

Rini et al. evaluated the efficacy of presurgical pazopanib in patients with localized RCC as a means to downsize the primary tumor and maximize the amount of renal parenchyma. This study is different compared to those previously discussed in that the goal was to perform partial nephrectomy in all patients, and these patients did not present with advanced disease. $25 \%$ of the patients in this study experienced a urine leak managed conservatively, while one patient had post-operative hemorrhage requiring embolization [34]. There was an overall high rate $(64 \%)$ of grade 3 complications, but no grade 4 or higher complications reported.

\section{Axitinib}

Axitinib is a potent VEGF and PDGF inhibitor [24]. A 2014 prospective study by Karam et al. evaluated a cohort of 24 patients who received axitinib prior to surgical intervention. A total of 19 patients underwent radical nephrectomy while the remaining 5 underwent partial nephrectomy. Similar to other studies, there was an increase in intraoperative adhesions appreciated in three patients, however none of which were prohibitive to the surgical intervention [15]. Post-operatively, two patients experienced grade 3 complications including chylous ascites requiring percutaneous drainage and postoperative bleeding requiring reoperation. One superficial wound dehiscence was appreciated and managed conservatively.

\section{CONCLUSIONS}

While the exact role of presurgical therapy prior to intervention in patients with advanced disease is not clearly defined, a continuing amount of literature is demonstrating the effect of these medications on surgical complications. While several studies have outlined specific complications including an increased in surgical adhesions, the remainder of the identified complications do not appear to be directly related to the use of therapy. Wound complications have been sporadically reported in the literature but do not appear to be consistent. Concern for an increased risk of surgical complications would not seem to be a primary concern in providing patients with presurgical therapy. As we enter into the era of immunotherapy, it will be critical to thoroughly understand the potential peri-operative risks posed by these new agents. Most concerning is the potential for severe autoimmune effects, including pancreatitis, pneumonitis, and neuropathy that may affect to post-operative recovery process [35]. The PROSPER study will examine patients undergoing nephrectomy with or without presurgical nivolumab. On-going trials such as these will be of particular importance as we seek to gain an understanding of their impact on surgical efficacy and complications. 


\section{REFERENCES}

[1] Siegel RL, Miller KD, Jemal A. Cancer statistics, 2017. CA: A Cancer Journal for Clinicians 2017;67(1):7-30.

[2] Thorstenson A, Bergman M, Scherman-Plogell AH, Hosseinnia S, Ljungberg B, Adolfsson $\mathrm{J}$, et al. Tumour characteristics and surgical treatment of renal cell carcinoma in Sweden 2005-2010: A population-based study from the national Swedish kidney cancer register. Scandinavian Journal of Urology 2014;48(3):231-8.

[3] Bazzi WM, Sjoberg DD, Feuerstein MA, Maschino A, Verma S, Bernstein M, et al. Long-term survival rates after resection for locally advanced kidney cancer: Memorial Sloan Kettering Cancer Center 1989 to 2012 experience. The Journal of Urology 2015;193(6):1911-6.

[4] Posadas EM, Limvorasak S, Figlin RA. Targeted therapies for renal cell carcinoma. Nature Reviews Nephrology 2017;13(8):496-511.

[5] Motzer RJ, Escudier B, McDermott DF, George S, Hammers HJ, Srinivas S, et al. Nivolumab versus everolimus in advanced renal-cell carcinoma. The New England Journal of Medicine 2015;373(19):1803-13.

[6] Escudier B, Tannir NM, McDermott DF, Frontera OA, Melichar B, Plimack ER, et al. LBA5CheckMate 214: Efficacy and safety of nivolumab + ipilimumab $(\mathrm{N}+\mathrm{I}) \mathrm{v}$ sunitinib (S) for treatment-naïve advanced or metastatic renal cell carcinoma (mRCC), including IMDC risk and PD-L1 expression subgroups. Annals of Oncology 2017;28(suppl_5):mdx440.029-mdx440.029.

[7] Flanigan RC, Salmon SE, Blumenstein BA, Bearman SI, Roy V, McGrath PC, et al. Nephrectomy followed by interferon alfa- $2 b$ compared with interferon alfa- $2 b$ alone for metastatic renal-cell cancer. The New England Journal of Medicine 2001;345(23):1655-9.

[8] Mickisch GH, Garin A, van Poppel H, de Prijck L, Sylvester R. Radical nephrectomy plus interferon-alfabased immunotherapy compared with interferon alfa alone in metastatic renal-cell carcinoma: A randomised trial. Lancet (London, England) 2001;358(9286):966-70.

[9] Motzer RJ, Jonasch E, Agarwal N, Bhayani S, Bro WP, Chang SS, et al. Kidney cancer, version 2.2017, NCCN clinical practice guidelines in oncology. Journal of the National Comprehensive Cancer Network: JNCCN 2017;15(6): 804-34.

[10] Hanna N, Sun M, Meyer CP, Nguyen PL, Pal SK, Chang SL, et al. Survival analyses of patients with metastatic renal cancer treated with targeted therapy with or without cytoreductive nephrectomy: A national cancer data base study. Journal of Clinical Oncology: Official Journal of the American Society of Clinical Oncology 2016;34(27):3267-75.

[11] Haas NB, Manola J, Uzzo RG, Flaherty KT, Wood CG, Kane C, et al. Adjuvant sunitinib or sorafenib for highrisk, non-metastatic renal-cell carcinoma (ECOG-ACRIN E2805): A double-blind, placebo-controlled, randomised, phase 3 trial. Lancet (London, England) 2016;387(10032): 2008-16.

[12] Ravaud A, Motzer RJ, Pandha HS, George DJ, Pantuck AJ, Patel A, et al. Adjuvant sunitinib in high-risk renal-cell carcinoma after nephrectomy. The New England Journal of Medicine 2016;375(23):2246-54.

[13] Motzer RJ, Haas NB, Donskov F, Gross-Goupil M, Varlamov S, Kopyltsov E, et al. Randomized phase III trial of adjuvant pazopanib versus placebo after nephrectomy in patients with localized or locally advanced renal cell carcinoma. Journal of Clinical Oncology: Official
Journal of the American Society of Clinical Oncology 2017:Jco2017735324.

[14] Jonasch E, Wood CG, Matin SF, Tu SM, Pagliaro LC, Corn PG, et al. Phase II presurgical feasibility study of bevacizumab in untreated patients with metastatic renal cell carcinoma. Journal of Clinical oncology: Official Journal of the American Society of Clinical Oncology 2009;27(25):4076-81.

[15] Karam JA, Devine CE, Urbauer DL, Lozano M, Maity T, Ahrar K, et al. Phase 2 trial of neoadjuvant axitinib in patients with locally advanced nonmetastatic clear cell renal cell carcinoma. European Urology 2014;66(5):874-80.

[16] Borregales LD, Adibi M, Thomas AZ, Wood CG, Karam JA. The role of neoadjuvant therapy in the management of locally advanced renal cell carcinoma. Therapeutic Advances in Urology 2016;8(2):130-41.

[17] Grossman HB, Natale RB, Tangen CM, Speights VO, Vogelzang NJ, Trump DL, et al. Neoadjuvant chemotherapy plus cystectomy compared with cystectomy alone for locally advanced bladder cancer. The New England Journal of Medicine 2003;349(9):859-66.

[18] Cost NG, Krabbe LM, Bagrodia A, Margulis V. The use of preoperative targeted molecular therapy to allow nephron sparing for T1b tumors. Current Opinion in Urology 2013;23(5):411-7.

[19] Chapin BF, Delacroix Jr SE, Culp SH, Nogueras Gonzalez GM, Tannir NM, Jonasch E, et al. Safety of presurgical targeted therapy in the setting of metastatic renal cell carcinoma. European Urology 2011;60(5):964-71.

[20] Choueiri TK, Motzer RJ. Systemic therapy for metastatic renal-cell carcinoma. The New England Journal of Medicine 2017;376(4):354-66.

[21] Escudier B, Pluzanska A, Koralewski P, Ravaud A, Bracarda S, Szczylik C, et al. Bevacizumab plus interferon alfa-2a for treatment of metastatic renal cell carcinoma: A randomised, double-blind phase III trial. Lancet (London, England) 2007;370(9605):2103-11.

[22] Rini BI, Halabi S, Rosenberg JE, Stadler WM, Vaena DA, Ou SS, et al. Bevacizumab plus interferon alfa compared with interferon alfa monotherapy in patients with metastatic renal cell carcinoma: CALGB 90206. Journal of Clinical Oncology: Official Journal of the American Society of Clinical Oncology 2008;26(33):5422-8.

[23] Zhang H, Huang Z, Zou X, Liu T. Bevacizumab and wound-healing complications: A systematic review and meta-analysis of randomized controlled trials. Oncotarget 2016;7(50):82473-81.

[24] Vogelzang NJ, Sternberg CN. Signal-transduction inhibitors in renal cell carcinoma. BJU International 2007;99(5 Pt B):1289-95.

[25] Eichelberg C, Vervenne WL, De Santis M, Fischer von Weikersthal L, Goebell PJ, Lerchenmuller C, et al. SWITCH: A randomised, sequential, open-label study to evaluate the efficacy and safety of sorafenibsunitinib versus sunitinib-sorafenib in the treatment of metastatic renal cell cancer. European Urology 2015;68(5): 837-47.

[26] Harshman LC, Yu RJ, Allen GI, Srinivas S, Gill HS, Chung BI. Surgical outcomes and complications associated with presurgical tyrosine kinase inhibition for advanced renal cell carcinoma (RCC). Urologic Oncology 2013;31(3): 379-85.

[27] Pattaras JG, Moore RG, Landman J, Clayman RV, Janetschek G, McDougall EM, et al. Incidence of postoperative adhesion formation after transperitoneal 
genitourinary laparoscopic surgery. Urology 2002;59(1): 37-41.

[28] Hellenthal NJ, Underwood W, Penetrante R, Litwin A, Zhang S, Wilding GE, et al. Prospective clinical trial of preoperative sunitinib in patients with renal cell carcinoma. The Journal of Urology 2010;184(3):859-64.

[29] Powles T, Kayani I, Blank C, Chowdhury S, Horenblas S, Peters J, et al. The safety and efficacy of sunitinib before planned nephrectomy in metastatic clear cell renal cancer. Annals of oncology: Official Journal of the European Society for Medical Oncology 2011;22(5):1041-7.

[30] Lane BR, Derweesh IH, Kim HL, O'Malley R, Klink J, Ercole CE, et al. Presurgical sunitinib reduces tumor size and may facilitate partial nephrectomy in patients with renal cell carcinoma. Urologic Oncology 2015;33(3):112. e15-21.

[31] Cowey CL, Amin C, Pruthi RS, Wallen EM, Nielsen ME, Grigson G, et al. Neoadjuvant clinical trial with sorafenib for patients with stage II or higher renal cell carcinoma. Journal of Clinical Oncology: Official Journal of the American Society of Clinical Oncology 2010;28(9):1502-7.

[32] Zhang Y, Li Y, Deng J, Ji Z, Yu H, Li H. Sorafenib neoadjuvant therapy in the treatment of high risk renal cell carcinoma. PLoS One 2015;10(2):e0115896.

[33] Powles T, Sarwar N, Stockdale A, Sarker SJ, Boleti E, Protheroe A, et al. Safety and efficacy of pazopanib therapy prior to planned nephrectomy in metastatic clear cell renal cancer. JAMA Oncology 2016;2(10):1303-9.

[34] Rini BI, Plimack ER, Takagi T, Elson P, Wood LS, Dreicer $\mathrm{R}$, et al. A phase II study of pazopanib in patients with localized renal cell carcinoma to optimize preservation of renal parenchyma. The Journal of Urology 2015;194(2):297-303.

[35] Elias AW, Kasi PM, Stauffer JA, Thiel DD, Colibaseanu DT, Mody K, et al. The feasibility and safety of surgery in patients receiving immune checkpoint inhibitors: A retrospective study. Frontiers in Oncology 2017;7:121. 